\title{
An Electrical Stimulator to Suppress the Nervous Pain in Diabetic Neuropathic Patients
}

\author{
J. Francis Felix Sindhuja, S. Chitra, K. Deepthi, M. Anisha, R. Madhan Balaji
}

\begin{abstract}
Peripheral neuropathy is an acute disorder in diabetic patients who are suffering a very agonized pain in their peripheral nerves. Symptomatic relief can be achieved by some analgesic pain killers. One of the best approaches is using an electrical stimulation method where the sensory nerve fibers get excited by the application of pulsed currents at the site of pain. This could block the pain signals from reaching the brain, followed either by the pain gate theory or opioid mechanism. This study primarily focuses the feedback principle where the stimulus is given with respective to the patient's skin intensity to avoid skin burning in therapy. The opioid mechanism has been proved scientifically that it provides long-lasting pain relief even after the stimulation. This paper documents the design and operation of feedback system which provide a constant output by varying the width of pulses. Thereby, the patient's pain as well as the sufferings of skin reactions upon stimulus is reduced. As this electrical nerve stimulation strategy is a simple, safe, non-pharmacological, cost-effective it proves to be a better alternative for pain relief.
\end{abstract}

Keywords : Diabetic peripheral neuropathy, Feedback principle, Frequency, Neuropathic pain.

\section{INTRODUCTION}

$\mathrm{D}_{\mathrm{i}}$ abetes is a serious, chronic disease that exists either when pancreas is inadequate to bring out enough insulin (Type 1), or when the body can't constructively handle the insulin it brings out (Type 2). Type 2 diabetes contributes most of the people with this disease throughout the world. If diabetes elongate overtime, can destruct the heart, blood vessels, eyes, kidneys and nerves, and increases the possibility of cardiac disease, renal failure, paralysis etc [1]. This contributes to the constricted blood flow in the feet. It further makes the damages which are unperceived, it go forward to sore, contaminations and the increased need of the replacement of the limb region. In diabetic patients about $60-70 \%$ develops peripheral neuropathy. The peripheral

Revised Manuscript Received on December 16, 2019.

* Correspondence Author

J.Francis Felix Sindhuja, Department of Biomedical Engineering, Kalasalingam Academy of Research and Education, Krishnankoil, India.

S.Chitra, Department of Biomedical Engineering, Kalasalingam Academy of Research and Education, Krishnankoil, India.

K.Deepthi, Department of Biomedical Engineering, Kalasalingam Academy of Research and Education, Krishnankoil, India.

Anisha M*, Department of Biomedical Engineering, Kalasalingam Academy of Research and Education, Krishnankoil, India.

Email: anisha@klu.ac.in

R.Madhan Balaji, Department of Biomedical Engineering, Agni College of Engineering, India. portion of the nervous system has the adverse effects by the peripheral neuropathy. The nerves on the peripheral region are accountable for the commotions like touch, pain, and temperature. In the hands and feet many highly strenuous nerves are available; this is a consequence of the requirement for sensory combination with numerous small muscles and diversity of fluctuations in these bodies [2]. The nerves and regions of the body that are affected by the neuropathy will determine the indications to the patient. Some indications of diffuse peripheral neuropathy include burning, excruciating, stabbing or tormenting or presented with tingling, savage, muscle weakness, loss of balance, numbness.

A person's standard of living including sleep, job, creativity, prosperity and social activities can be affected. Small nerve fiber damage contributes to agonizing neuropathy with protection of active large medullated fibers [3]. Commonly, the damaged large nerve fibers, contributes to motor and proprioceptive function loss. Blood supply to the nerves can be affected by inflamed blood vessels that injuries the nerves due to the angititis [4]. These indications of physiopathology are due to primarily metabolic and vascular factors. There is a rapid improvement in sorbitol and fructose, glycated end-products, reactive oxygen species and triggering of protein kinase-C. All the above elements contribute to damage of the nervous system and the inactivation effectively [5].

Today diabetic neuropathy can't be treated by any treatments; there is only a pain killer drugs to get relief from the symptoms. Some non-pharmacological therapies such as electrical stimulations being suppress the pain and discomfort associated with peripheral neuropathy. Since 2500 BC, Electric catfish and electric rays which can generate 300-volt shocks were used by the Ancient Egyptians to alleviate the pain [6]. The invention of electrostatic machines paves the way to be catalyzed the use of electrotherapeutic devices. As electrical stimulators are non-invasive, portable and non-pharmaceutical device, this ensures that it could be the best approach for treating the pain in neuropathic patients. 


\section{METHODOLOGY}

Transcutaneous electrical nerve stimulation (TENS) is a method which is used to get a pain relief. In this a low frequency current is applied between two electrode pads placed on the pain region. This applied electric charges flows through the area which is affected by neuropathy in addition it will activates the inactive nervous tissue, which will cause the analgesic result for the patient. A commonly used TENS is a battery operable unit which comprises of oscillator, step-down transformer, and frequency controller.

\section{A. theory of pain}

Melzack and Wall were developed the 'gate control theory' in 1960. They proposed that non- painful signals blocks the gates to painful signal, which prevents the realization of pain from the spinal cord. This contributes to the activation of the A-beta fibers, and with the help of this it will reduce the passing of the noxious (pain) stimulus from the ' $c$ ' fibers, through the spinal cord and hence on to the higher $\mathrm{c}$ fibers. At a relatively high rate (in the order of 90-130 $\mathrm{Hz}$ or pps) A-beta fibers are getting activated. Because of this activation,on the dorsal root fibers presynaptic inhibition can be implemented which conjugates on nociceptors spinal neurons. The incoming noxious painful information will be stopped from reaching the CNS with the help of inhibition of presynapses.

\section{B. Opioid Mechanism}

An another theory associated with the relief of symptomatic pain is an "opioid mechanism' which constitutes the activation of the A-delta fibers which will give response preferably to a low frequency stimulation $(2-10 \mathrm{~Hz})$, will activate the natural pain killers called opioids, this will give relief from pain by the use of endogenous opiate (encephalin) in the spinal cord for the suppressing of the noxious sensory pathway activity. Many CNS functions, including respiration, food intake, sexual activity, learning, decision making and memory can be influenced by the Opioidergic neurotransmission in the spinal cord and in brain. Beta-endorphins, enkephalins and the dynorphins are the three main classifications of endogenous opioids. These opioids are having the ability to change the nociceptive input in the following ways:Obstructing the epinephrine ransom by hindrance of $\mathrm{Ca} 2+$ influx within the presynaptic membrane.

1) Obstructing the epinephrine ransom by hindrance of $\mathrm{Ca} 2+$ influx within the presynaptic membrane.

2) Launching of potassium channels $(\mathrm{K}+)$, where hyper polarizations of neurons take place.

To produce the analgesic effect, the most important CNS sites such as the periaqueductal gray (PAG), nucleus raphe magnus (NRM), dorsal raphe(DR), caudate nucleus(CN), septalnucleus ( $\mathrm{Spt}$ ) are to be stimulated which is important to the suppression of pain pathway to obstruct the noxious incoming signal at spinal cord. And these CNS segments consist of the improved densities of analgesic receptors. The pain due to the neuropathy is obstructed by this stimulation, that is, it makes the analgesics, while the touch, pressure, and temperature commotion remain inviolate. This type of mechanism used for a longer time after excitation given to the patient. In laminae I, II and V, electrical excitation of PAG or NRM obstructs the spinal thalamic cells so that incoming signals from the nociceptors are modified at the CNS level. PAG and NRM are connected by neuronal passage. The usage of PAG generally exists by the excitation of the inclining passage way from NRM and most commonly by excitation of ascending connections lies on sub cortical regions of the CNS. The opiate receptors gets activated at the inter neuronal level which creates neuron hyper polarization that results in the obstruction of triggering and delivers the substance $P$ which is constitutes a neurotransmitter which is responsible in pain transmission, causes the blockage of pain signals. This will make the release of endorphin and is obstructed by the opiates. During this excitation of these opiates, serotonin (5-HT) is also free from axons in subcortical nuclei and in spinal cord in CNS. This modifies the passage of pain by obstructing the incoming signals.

\section{Frequency Selection for Therapy}

The number of electrical pulses is to be delivered to the body within one second is termed as Frequency. The pulses are delivered by the oscillator in the form of electric charges at rate of about 1 or 2 pulses per second (pps) up to 200 or 250 pps. Most commonly $2-150 \mathrm{Hzrange}$ of frequency is used for getting pain relief in an efficient manner. Frequency selection for therapy is depicted in Figure 1. The intensity of current will commonly in the range of about 0 $80 \mathrm{~mA}$, though $100 \mathrm{~mA}$ intensity of output signal can be obtained from some form of TENS unit. As our aim target region for the treatment is the sensory peripheral nerves, which is to be stimulated at a small intensity of charges, thus the release of these small amount of charges are much adequate for the nerve to depolarize, to get the modality efficient way. The pulse duration can be varied from about 40 to 250 micro seconds ( $\mu \mathrm{s})$. Short duration of pulses is most preferable for low threshold value and also it will be responsible for a change of states. There is generally no requirement of a long duration pulse to insist the depolarization of nerves, thus the excitation for a millisecond is well enough for the TENS treatment.

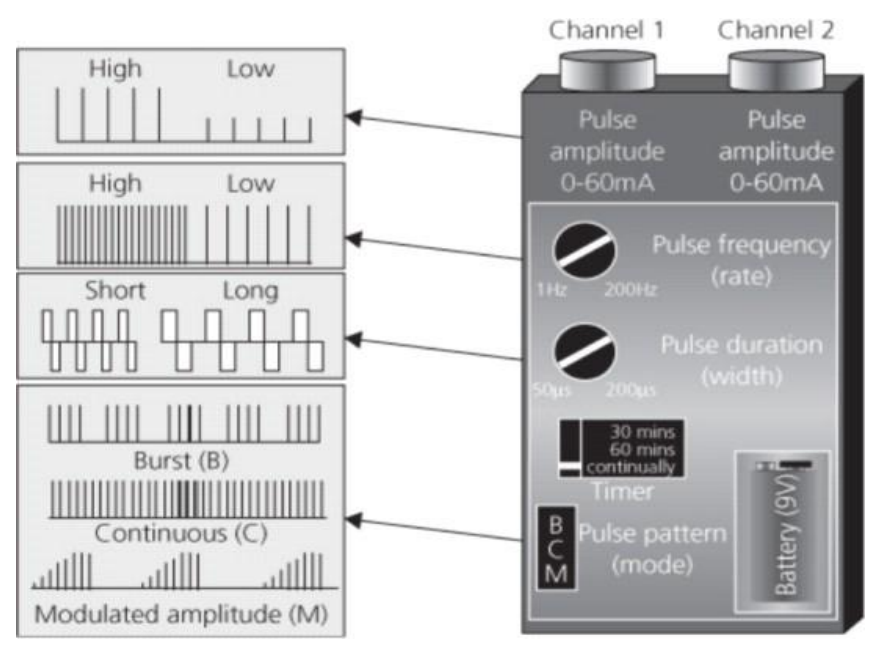

Fig.1 Frequency Selections for Therapy 
Depending on the production of a TENS unit the pulsing modes can be varied, but it constitutes to the biphasic square wave pulses in an assymetrical mode. The biphasic pulse do not allow DC component through it, thus approximating and reducing the skin infections due to the creation of electrolytes beneath the electrodes placed on the skin. Low frequency stimulation of about $2-5 \mathrm{~Hz}$ with longer duration of pulses $(200-250 \mu \mathrm{s})$ is very efficient. . 30 minutes duration will adequate for a minimum dose. In TENS device there is a requirement of some period of time to create the natural pain killers and hence the onset of pain relief may be slower. Once the adequate opioids has been delivers at the segment. However, it will tend to work after the given excitation. Most of the neuropathic patients handled that at the minimum range of excitation. In this proposed paper, effectiveness of the low frequency type TENS method is discussed. Block Diagram of the proposed approach is shown in Figure 2.

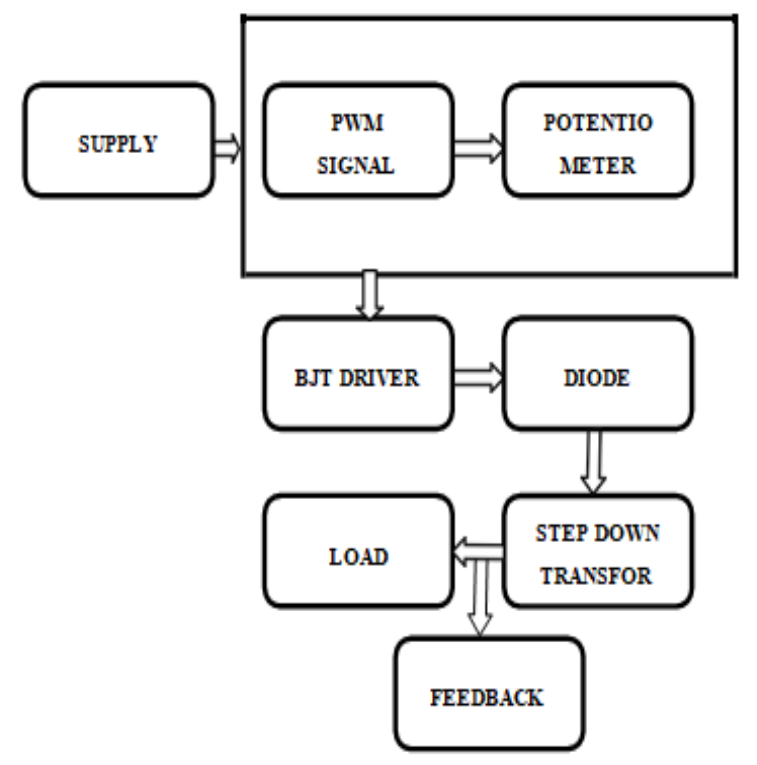

Fig .2.Block Diagram of Proposed Approach

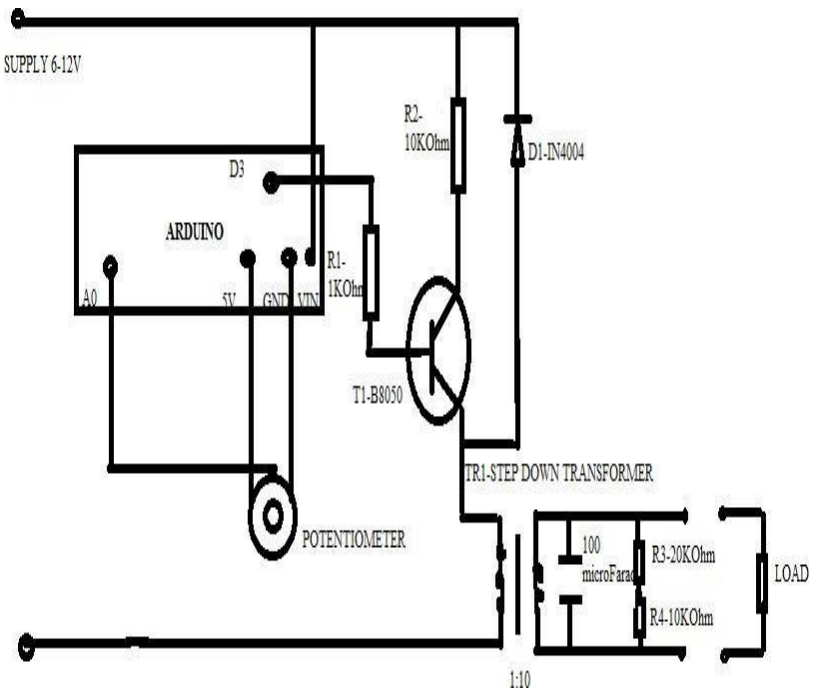

Fig .3.Circuit Diagram Without Feedback Loop

The increment or decrement in the voltage taken place in the above circuit and it keeps the voltage stable for the load. Discharging time of the output can be changed by changing the load terminal value. High rate of current is passing for low value of load terminal, and so it can changes the duty cycle and discharging time. If we add a feedback loop that sense the patients intensity with the above circuit and gives it to the PWM pin on the Arduino for the desired value with respect to the patient. Circuit diagram of TENS without and with feedback loop has been shown in Figure $3 \& 4$ respectively.

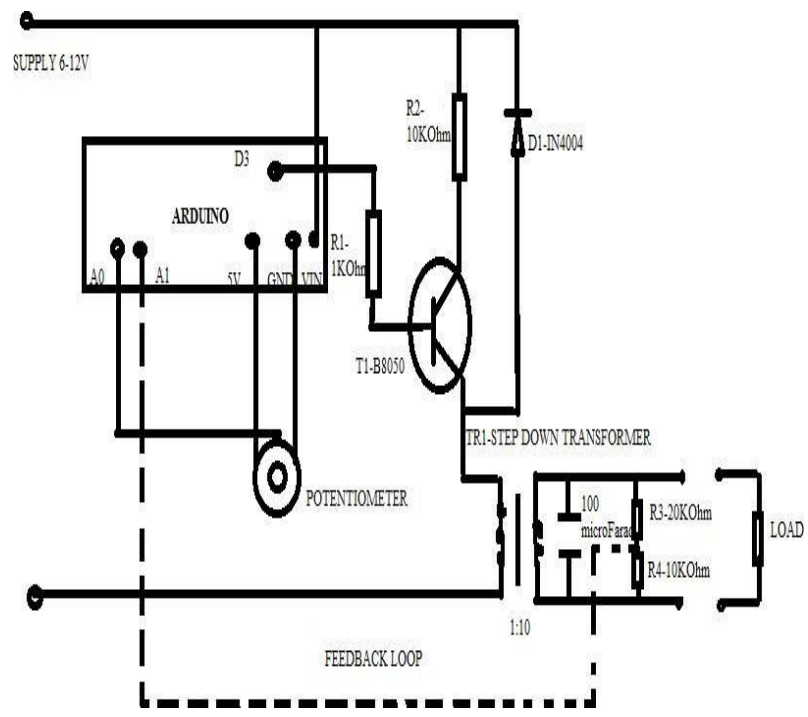

Fig .4. Circuit Diagram with Feedback Loop

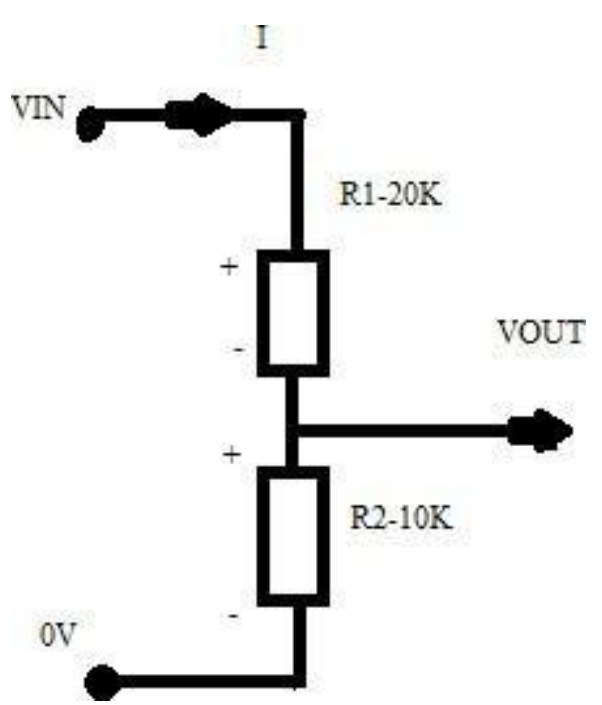

Fig .5. Feedback Resistance

When the anode voltage is lesser than the cathode, the switch tends to close. The flow of charges will start to improve, and opposing voltage fluctuations can be created by inductor among its terminals according to the fluctuations in the currents. It creates the voltage drop across it and reduces the total amount of voltage across the output. In another condition the switch is tends to be opened while the varying current is present, the output voltage will be less than the input. If the switch is tends to open repeatedly, there will be a decrement in the current value. This creates the voltage drop which acts as a current supply. 


\section{RESULTS AND DISCUSSION}

It is evident that for the treatment of the diabetic peripheral neuropathy TENS is a safe and efficient way of cure. In addition to that it encourages the usage of TENS as the wearable stimulator. The pain inhibition can be effectively achieved by TENS rather than any other devices. The endothelial growth factor will get increased by the use of this device. The electrical stimulation given by this device has the ability to excite the large myelinated nerves.

By giving connections as per circuit diagram, shown in Figure 5, it tends to acquire the pulsed constant output waveform. Circuit connection is shown in Figure 6. As per the Arduino coding, the establishment of feedback principle is to be succeeded as we gaining the constant output values by changing the voltage in potentiometer. This results shows that with respective to the patients skin intensity, the delivering of electric pulses are modulated as a desired value.

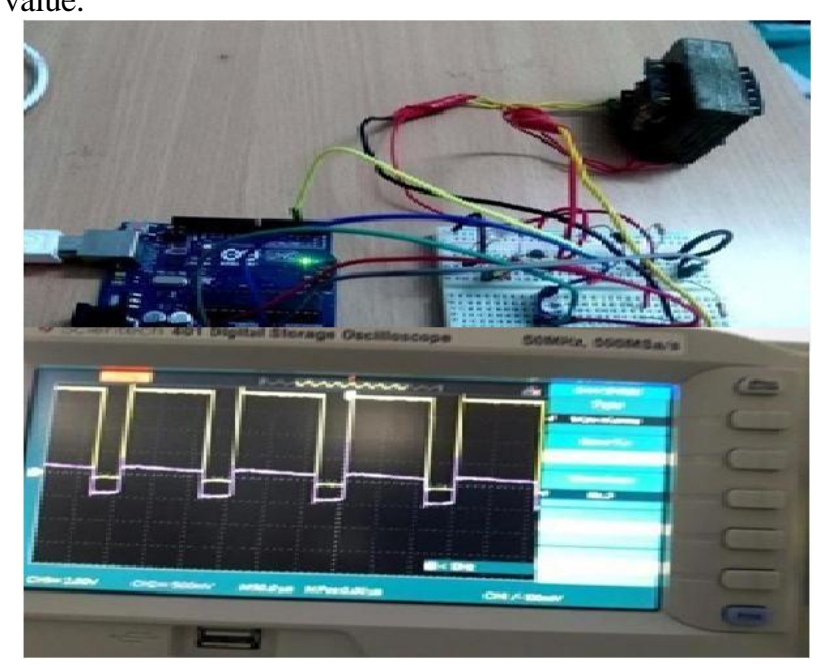

Fig .7. Output Waveform

The obtained output waveform has been shown in Figure 7. The result shows the possibility of suppressing severe chronic is markedly related to the frequency of conditional stimulation. And the feedback technique can make a best approach for giving safe therapy.

\section{CONCLUSION}

In this approach, the facility of feedback system is made, where the given input frequency has to be adapted with the patient's pain intensity. Thus, it can reduce the severity arising from existing TENS device. For the treatment of symptomatic diabetic peripheral neuropathy, TENS is a best non pharmacological and safer method of stimulation. There is a great need for reducing side effects of medicine which is often used by many people. Thus this implementation is cost effective and simple, will be a very great and useful setup in medical field.A conclusion section is not required. Although a conclusion may review the main points of the paper, do not replicate the abstract as the conclusion. A conclusion might elaborate on the importance of the work or suggest applications and extensions.

\section{REFERENCES}

1. Ayesha Girach, Thomas Henry Julian, Giustino Varrasi, Antonella Panagiotis. Quality of Life in Painful Peripheral Neuropathies :A Systemic Review, Hindawi,233 May 2019.
2. P.zia , P.G.Sarrigiannis, D.Rao,C. Hewamadduma, M.Hadjivassilliou, "Chronic Idiopathic axonal Polyneuropathy: prevelance of pain and impact on quality of life," Scopus, 2019.

3. AnetaDemidas and mikolaj, zaezycki, :Touch and pain sensations in diadynamic current(DD) and Transcutaneous Electrical Nerve Stimulation(TENS) :A randomized study",17 April 2019.

4. A. K.Artermiadis and P.Zis, "Neuropathic pain in acute and sub-acute neuropathies: A systemic review", Google scholar, 2018.

5. Casey A.grover, Mia potter Mc Kernan, Reb J.H. Close et al. "TENS in the emergency department for pain relief: A preliminary study of efficacy and feasibility", Western Journal of Emergency Medicine and Integrating Emergency Care Population Health,9 August 2018.

6. Agamohammadi, D., Farzin, H., Fakhari, S.and Siami.R, "The E valuation of the effect of TENS therapy on reducing acute and chronic pain following varicocelectomy", Open Journal of Medicine, 7(03),p.52

7. Neurometrics Inc., Waltham, MA, USA et al. "Fixed-site high frequency TENS for treatment of chronic low back and lower extremity pain", Dove press journal, 28 June 2016.

8. Mr. Sathriya Pranatha, Dr. Heri Nugroho, and Dr. Untung Sugianto et al. "The effect of TENS towards pain level of patients with diabetes mellitus with peripheral neuropathy in diabetic foot ulcer treatment in Yogyakarta general hospital Indonesia”, IOSR journal of nursing and health service (IOSR-JNHS), 5 September 2016.

9. Patricia Branco Mills, MD, MHSc, FRCPC, Farhana Dossa BASc, and MSc. et al. "TENS for management of limb spasticity", Wolters Kluwer Health Inc, 4 April 2016.

10. Igwea, S.E.,Tabansi-Ochuogu,C.S. and Abaraogu,U.O. "TENS and heat therapy for pain relief and quality of life improvement in individuals with primary dysmenorrheal" :A Systemic review complementary therapies in clinical practice, $24, \mathrm{pp}, 86-91$. 